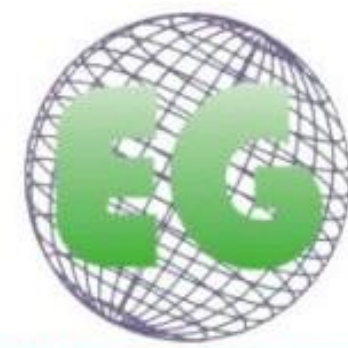

ISSN 1695-6141 $N^{\circ} 49$

\title{
Evaluación de los Ensayos Clínicos Aleatorios desarrollados por enfermeras según los criterios de la Declaración CONSORT
}

Evaluation of randomized clinical essays developed by nurses according to the consort declaration criteria

\author{
Guzmán-Mendoza Edith ${ }^{1}$ \\ Santos-Díaz María de la Luz ${ }^{1}$ \\ Cabañas-Benitez Gisela ${ }^{1}$ \\ Pérez-Calderón Dulce María ${ }^{1}$ \\ Chávez-Aguilar Celsa ${ }^{1}$ \\ Morales-Rodríguez María Claudia ${ }^{2}$ \\ Landeros-Olvera Erick Alberto ${ }^{3}$ \\ ${ }^{1}$ Licenciada en Enfermería, Benemérita Universidad Autónoma de Puebla. México. \\ 2 Maestra en Ciencias de Enfermería, profesora investigadora, integrante del Cuerpo Académico \\ Intervenciones de Enfermería, Benemérita Universidad Autónoma de Puebla.México. \\ 3 Doctor en Ciencias de Enfermería, Integrante del Sistema Nacional de Investigadores, profesor \\ investigador de tiempo completo, integrante del Cuerpo Académico Intervenciones de Enfermería, \\ Benemérita Universidad Autónoma de Puebla. México.
}

\section{E-mail: erick landeross@hotmail.com}

\section{http://dx.doi.org/10.6018/eglobal.17.1.276091}

Recibido: $24 / 11 / 2016$

Aceptado: 23/02/2017

\section{RESUMEN:}

Objetivo: Evaluar si los Ensayos Clínicos Aleatorizados (ECAS) realizados por enfermeras en los últimos siete años, cumplen con la rigurosidad metodológica establecida por los criterios de la Declaración CONSORT.

Métodos: Revisión integrativa de la literatura de revistas de enfermería, se analizaron 66 ECAS realizados por enfermeras y publicados en 11 revistas indizadas en bases de datos reconocidas, en cuyo título, palabras clave o diseño se reconocieran como un ensayo clínico aleatorio. Se realizó un instrumento de 48 ítems para evaluar las características de los 66 ECAS publicados. Dicho instrumento está dividido en dos segmentos: el primero evalúa información general de los artículos y el segundo incluye las características de la Declaración CONSORT.

Resultados: El $57.6 \%$ de los ECAS utilizaron grupo control equivalente, $87.9 \%$ utilizó muestreo aleatorio, $28.8 \%$ enmascaramiento, $54.5 \%$ presentaron diagrama de flujo, $83 \%$ realizaron aleatorización, $57.6 \%$ describen las intervenciones empleadas a los grupos de estudio.

Conclusiones: De forma general, los ECAS publicados por enfermería en los últimos siete años no cumplen con los criterios de la Declaración CONSORT. Estos hallazgos representan un área de oportunidad para que editores de revistas de enfermería soliciten a los autores mayor apego a la rigurosidad metodológica en sus artículos de acuerdo a los criterios de la Declaración CONSORT.

Palabras clave: Evaluación; Ensayo Clínico; Investigación en Enfermería. 


\begin{abstract}
:
Objective: Evaluate if the Randomized Clinical Trials (RCTs) carried out by nurses in the last seven years fulfill the methodological rigor established by the CONSORT declaration criteria.

Methods: Nursing journals literature integral review; sixty-six RCTs carried out by nurses and published in 11 indexed journals within recognized data bases were analyzed and where their title, key words or design allowed them to be recognized as a randomized clinical essay. A 48 item instrument was realized to evaluate the characteristics of the 66 published RCTs. Such instrument is divides in two segments: the first one evaluates general information in the articles, and the second one includes the CONSORT Declaration characteristics.

Results: The $57.6 \%$ of RCTs used an equivalent control group, $87.9 \%$ used randomized sampling, $28.8 \%$ blind, $54.5 \%$ presented flow chart, groups.

Conclusions: In general, the RCTs published by nursing in the last seven years do not fulfill the CONSORT Declaration criteria. These findings present an opportunity area so nursing journals publishers request from the authors the most attachment to the methodological rigor in their articles, according to the CONSORT Declaration criteria.
\end{abstract}

Keywords: Evaluation; Clinical Trial; Nursing Research.

\title{
INTRODUCCIÓN
}

Los Ensayos Clínicos Aleatorios (ECAS) son experimentos controlados que regularmente se utilizan para evaluar la seguridad y eficacia de tratamientos 0 intervenciones ${ }^{(1)}$. Los ECAS bien diseñados y correctamente ejecutados, proporcionan la mejor evidencia sobre el efecto de las intervenciones sanitarias ${ }^{(2)}$. Sin embargo, la rigurosidad metodológica para ejecutar un experimento controlado con grupos paralelos, no siempre se lleva a cabo. Diversos grupos de investigadores se han interesado en identificar cuáles son los procedimientos sistemáticos para llevar a cabo esta experimentación y obtener resultados confiables y poder realizar réplicas en otros contextos o poblaciones ${ }^{(3)}$. La evaluación de la calidad de los estudios científicos puede ser considerada esencial en el proceso de producción y selección de la literatura científica en la salud ${ }^{(4)}$. La guía más representativa fue publicada en 1996 denominada Normas Consolidadas para las Publicaciones de los Ensayos Clínicos (CONSORT por sus siglas en inglés), con la finalidad de unificar los criterios para publicar ECAS. Los criterios de la Declaración CONSORT han sido modificados constantemente para afinar los detalles metodológicos del diseño y los procedimientos a través de una lista de comprobación y un diagrama de flujo. La lista consta de 25 puntos específicos para comunicar los resultados o evaluar informes de ECAS de dos o más grupos paralelos ${ }^{(5,6)}$.

Respecto a la literatura publicada, no existe un trabajo reciente que evalúe si los ECAS realizados en ciertas disciplinas cumplen con los criterios de la Declaración CONSORT, al parecer se da por hecho que los ensayos publicados han sido evaluados por un comité editorial con base en los criterios de la Declaración CONSORT. La evaluación realizada a los ECAS publicados en la Revista Española de Anestesiología y Reanimación concluyó sobre una falta de datos relevantes sobre la ejecución de los ensayos así como una baja calidad metodológica de acuerdo a los criterios actuales $^{(7)}$, resultados similares reportan que la evaluación de los ECAS publicados en revistas biomédicas Chilenas, poseen serias deficiencias en los ensayos evaluados, pero es difícil saber si esas inconsistencias se deban a informes incompletos o a los diseños metodológicamente pobres ${ }^{(8)}$.

La presencia de estas debilidades metodológicas es bien conocida por los editores de las revistas científicas, quienes indican que los sesgos de los ECAS limitan la 
información disponible y condicionan el conocimiento, lo que afecta de manera particular a la medicina basada en la evidencia ${ }^{(9)}$.

En el caso de la disciplina de Enfermería, los ECAS representan una de las mejores fuentes de evidencia científica para mejorar los procedimientos del cuidado, tanto en las comunidades, familias, hospitales y práctica independiente ${ }^{(10,11)}$. No obstante, las publicaciones de enfermería con diseño experimental son mínimas, luego entonces es oportuno que sean evaluadas con la rigurosidad marcada por los criterios de la Declaración CONSORT.

Trabajos que evalúen los ECAS desarrollados por enfermería no han sido publicados, un estudio realizó un análisis de 358 estudios de enfermería publicados en revistas de México e identificaron que el $14 \%$ de los diseños de los artículos publicados son experimentales. Los autores no especifican las características metodológicas de este $14 \%$ o si se tratan de ECAS, solo señalan de manera general debilidades metodológicas como el muestreo no aleatorio, muestras pequeñas, instrumentos que se desconoce su confiabilidad y pruebas estadísticas mal empleadas ${ }^{(12)}$.

Ante la evidencia hasta ahora publicada, se plantea el siguiente propósito: Evaluar si los ECAS realizados por enfermeras en los últimos siete años, cumplen con la rigurosidad metodológica establecida por los criterios de la Declaración CONSORT.

\section{METODOLOGÍA}

Se trata de una revisión integrativa de la literatura, de revistas de enfermería través de la Biblioteca Virtual de la Benemérita Universidad Autónoma de Puebla. Los criterios de búsqueda fueron los siguientes: revistas indizadas (en bases de datos SciElo, EBSCO, CINAHL, CUIDEN, LILACS, Medline, PubMed y Thomson Reuters), artículos de investigación publicados del primer semestre del 2008 al segundo semestre del 2014, idiomas español, inglés y portugués, artículos en cuyo título, palabras clave o diseño se reconocieran como un ensayo clínico aleatorio.

Se analizaron 87 revistas en el periodo señalado, la producción fue de 9616 artículos, de éstos se seleccionaron 66 investigaciones por cumplir los criterios de inclusión, localizados en 11 revistas. Para observar el proceso de selección, véase la Figura 1. 
Figura 1. Diagrama de flujo del proceso de selección

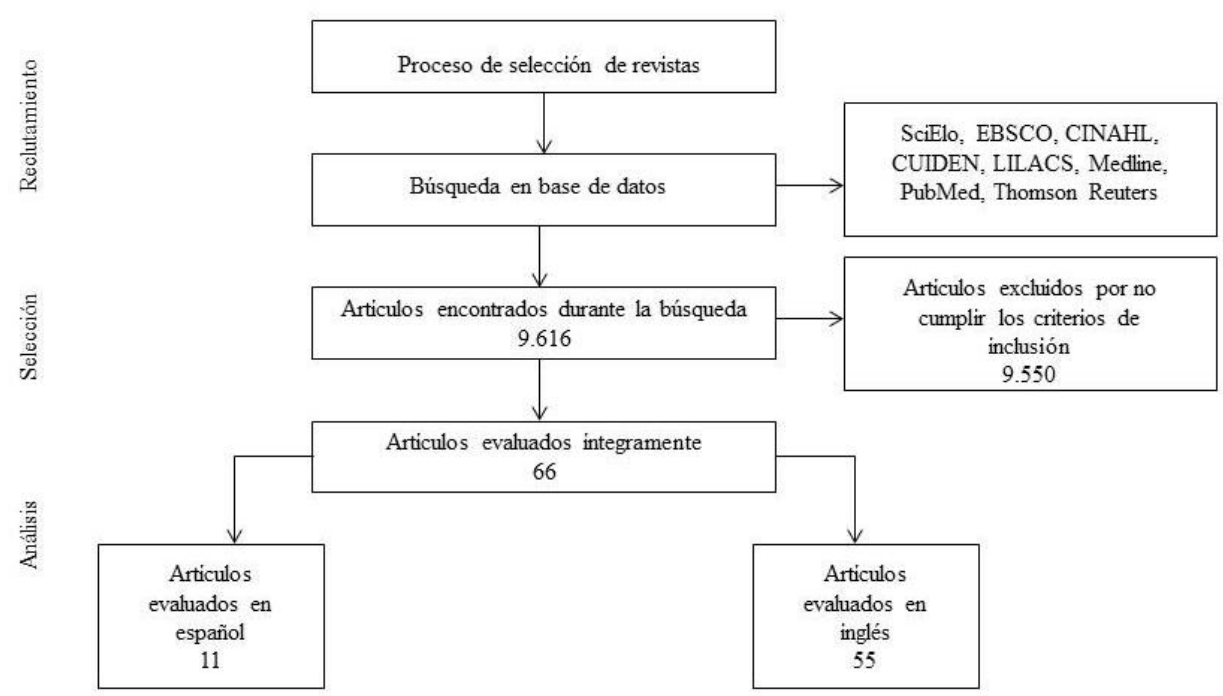

Se realizó un instrumento de 48 ítems para evaluar las características de los 66 ECAS publicados. Dicho instrumento está dividido en dos segmentos: El primero evalúa información general de los artículos (seis ítems) y el segundo incluye las características de la Declaración CONSORT. El instrumento fue piloteado y la revisión final se efectuó en un periodo de 90 días, cada artículo se revisó dos veces por investigadores diferentes para cotejar resultados y disminuir el sesgo. Este procedimiento se realizó a partir de la lectura completa de los artículos, focalizando la atención en los reactivos que el instrumento evaluó.

Consideraciones éticas: El protocolo y procedimientos de éste trabajo fueron evaluados para su registro en la Coordinación de Investigación de Pregrado de la Benemérita Universidad Autónoma de Puebla, México, bajo el número de referencia A-2015-0111-CIP. Análisis estadístico: El análisis de resultados se realizó con estadística descriptiva (frecuencias y porcentajes) a través del programa SPSS versión 21.

\section{RESULTADOS}

Se analizaron 66 ECAS de enfermería publicados en el periodo del 2008 al 2014 en revistas indizadas de habla hispana e inglesa. Los 66 ECAS analizados provienen de España (6), Colombia (3), México (3), Brasil (2), Turquía (2), EEUU (30), Corea del Sur (2), Australia(2), Irán (6), China (2), Canadá (1), Portugal (1), Países Bajos (1) y los que no especifican lugar de origen (5).

Los hallazgos descriptivos fueron los siguientes: 34 ECAS $(51.5 \%)$ muestran como primer autor a un doctor en ciencias de enfermería, masters en 13 ECAS (19.7\%), especialistas y licenciados en una publicación (1.5\% respectivamente), mientras que en 17 ECAS (25.7\%) no se mencionan.

Los lugares de procedencia donde se realizaron los ECAS, muestran que 38 (57.6\%) son de universidades, 22 (33.3\%) de instituciones de salud, mientras que 6 (9\%) restantes se divide en clínicas e institutos de investigación. Respecto a la colaboración, 25 ECAS (37.9\%) son multidisciplinarios, el resto son exclusivamente realizados por profesionales de enfermería. 
La principal temática que prevaleció fue la clínica con 46 ECAS (69.7\%), educativo con 17 ECAS $(25.8 \%)$ y la temática familiar entre otros temas lo muestran 3 ECAS (4.5\%).

Los hallazgos en cuanto a los criterios de la Declaración CONSORT se muestran en la siguiente tabla:

Tabla I. Resultados de los criterios: Declaración CONSORT. Puebla México, 2014.

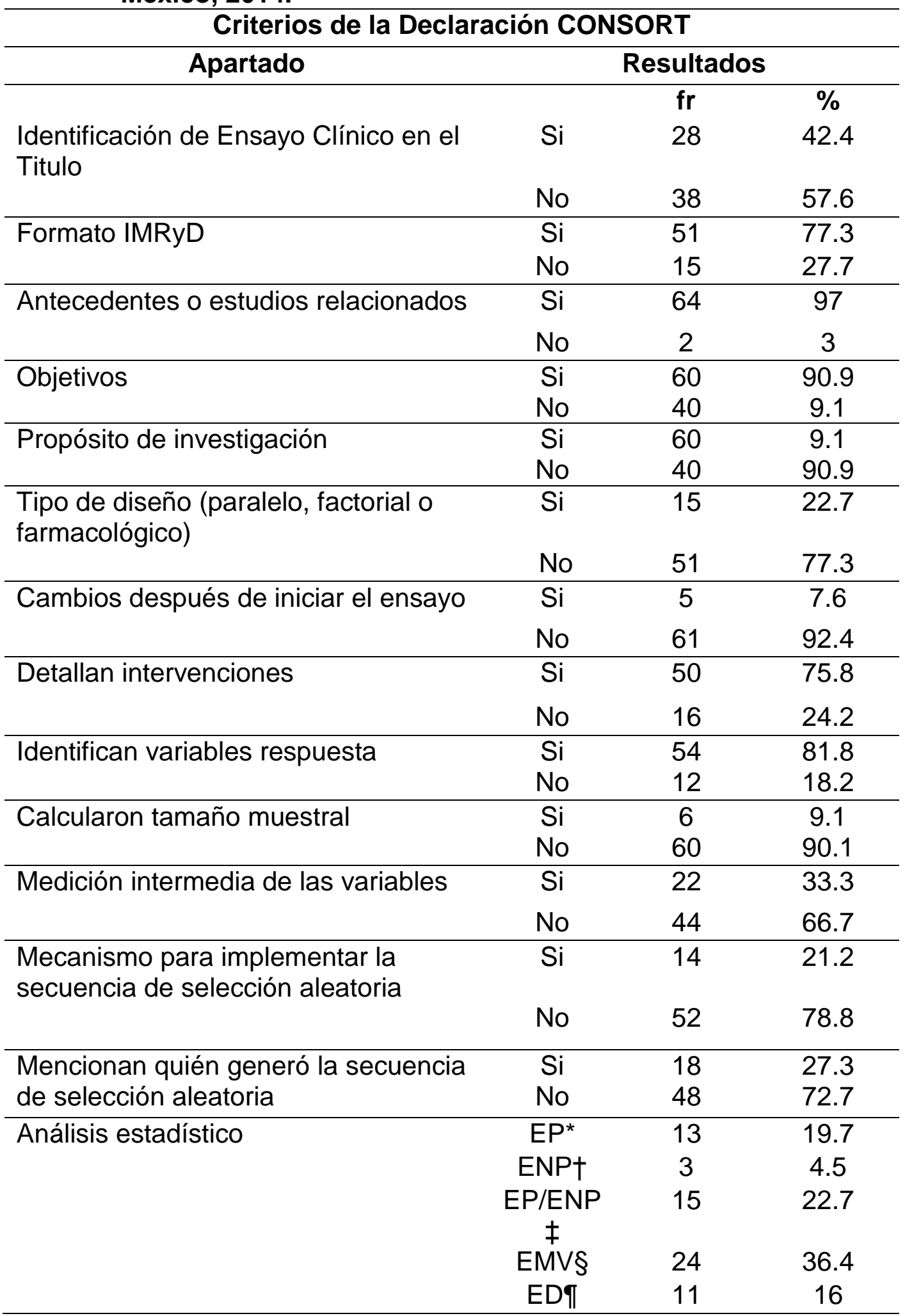




\begin{tabular}{|c|c|c|c|}
\hline \multirow{2}{*}{$\begin{array}{l}\text { Análisis adicionales (subgrupos y } \\
\text { ajustados) }\end{array}$} & $\mathrm{Si}$ & 55 & 83.3 \\
\hline & No & 11 & 16.7 \\
\hline \multirow{2}{*}{$\begin{array}{l}\text { Diagrama de flujo con número de } \\
\text { participantes para cada grupo }\end{array}$} & Si & 31 & 47 \\
\hline & No & 35 & 53 \\
\hline \multirow{2}{*}{$\begin{array}{l}\text { Mencionan el número de participantes } \\
\text { excluidos }\end{array}$} & $\mathrm{Si}$ & 31 & 47 \\
\hline & No & 35 & 53 \\
\hline \multirow{2}{*}{$\begin{array}{l}\text { Menciona fechas de reclutamiento y } \\
\text { seguimiento de los participantes }\end{array}$} & $\mathrm{Si}$ & 29 & 43.9 \\
\hline & No & 57 & 56.1 \\
\hline \multirow{2}{*}{$\begin{array}{l}\text { Indican causa externa para finalizar o } \\
\text { interrumpir el estudio }\end{array}$} & $\mathrm{Si}$ & 9 & 59.1 \\
\hline & No & 27 & 40.9 \\
\hline \multirow{2}{*}{$\begin{array}{l}\text { Presentan tablas de datos } \\
\text { sociodemográficos y clínicos de la } \\
\text { población de estudio }\end{array}$} & $\mathrm{Si}$ & 53 & 80.3 \\
\hline & No & 13 & 19.7 \\
\hline \multirow{2}{*}{$\begin{array}{l}\text { Detallan el número de participantes en } \\
\text { cada análisis }\end{array}$} & $\mathrm{Si}$ & 53 & 80.3 \\
\hline & No & 13 & 19.7 \\
\hline \multirow{2}{*}{$\begin{array}{l}\text { Mencionan resultados para el grupo } \\
\text { control e intervención }\end{array}$} & $\mathrm{Si}$ & 56 & 84.8 \\
\hline & No & 10 & 15.2 \\
\hline \multirow[t]{2}{*}{ Mencionan IC $\geq 95 \%$} & $\mathrm{Si}$ & 56 & 84.8 \\
\hline & No & 10 & 15.2 \\
\hline \multirow[t]{2}{*}{ Calcularon tamaño del efecto absoluto } & $\mathrm{Si}$ & 17 & 25.8 \\
\hline & No & 49 & 74.2 \\
\hline \multirow[t]{2}{*}{ Calcularon tamaño del efecto relativo } & $\mathrm{Si}$ & 4 & 6.1 \\
\hline & No & 62 & 93.9 \\
\hline \multirow[t]{2}{*}{ Muestran resultados de otro análisis } & $\mathrm{Si}$ & 4 & 6.1 \\
\hline & No & 62 & 93.9 \\
\hline \multirow{2}{*}{$\begin{array}{l}\text { Indican haber ocasionado un daño o } \\
\text { perjuicio a los participantes del estudio }\end{array}$} & $\mathrm{Si}$ & 27 & 40.9 \\
\hline & No & 39 & 59.1 \\
\hline \multirow{2}{*}{$\begin{array}{l}\text { Indican haber ocasionado un daño o } \\
\text { perjuicio a los participantes del estudio }\end{array}$} & $\mathrm{Si}$ & 27 & 40.9 \\
\hline & No & 39 & 59.1 \\
\hline \multirow[t]{2}{*}{ Señalan limitaciones del estudio } & $\mathrm{Si}$ & 10 & 15.2 \\
\hline & No & 56 & 84.8 \\
\hline \multirow{2}{*}{$\begin{array}{l}\text { Refieren la posibilidad de generalizar } \\
\text { el resultado de su ensayo (validez } \\
\text { externa) }\end{array}$} & $\mathrm{Si}$ & 45 & 68.2 \\
\hline & No & 21 & 31.8 \\
\hline \multirow{2}{*}{$\begin{array}{l}\text { Aplican una interpretación consistente } \\
\text { con sus resultados }\end{array}$} & $\mathrm{Si}$ & 34 & 51.5 \\
\hline & No & 32 & 48.5 \\
\hline
\end{tabular}




\begin{tabular}{lccc}
\hline Presentan número de registro & $\mathrm{Si}$ & 49 & 74.2 \\
& $\mathrm{No}$ & 17 & 25.8 \\
\hline Presentan número de registro ante un & $\mathrm{Si}$ & 13 & 19.7 \\
comité de ética & $\mathrm{No}$ & 53 & 80.3 \\
\hline Mencionan consentimiento informado & $\mathrm{Si}$ & 42 & 84 \\
& $\mathrm{No}$ & 24 & 16 \\
\hline Mencionan prueba piloto & $\mathrm{Si}$ & 16 & 24.2 \\
& $\mathrm{No}$ & 50 & 75.8 \\
\hline Fueron financiados & $\mathrm{Si}$ & 9 & 13.6 \\
& $\mathrm{No}$ & 57 & 86.4 \\
\hline
\end{tabular}

IMRyD= Introducción, Metodología, Resultados y Discusión IC=Intervalos de Confianza

$\mathrm{EP}^{*}=$ Estadística Paramétrica; ENP†= Estadística No Paramétrica; EP/ENP $\neq=$ Estadística Paramétrica y Estadística No Paramétrica; $E M V \S=$ Estadística Multivariada; ED $=$ Estadística Descriptiva.

En el mismo orden de ideas, las características principales de un ECA desde el punto de vista de su arquitectura metodológica es mostrar: 1) Muestreo aleatorio, 2) al menos dos grupos paralelos o equivalentes para la contrastación estadística, 3) que sean descritas las intervenciones que se realizaron al grupo(os) control, 4) que las intervenciones sean enmascaradas o ciegas y 5) que el artículo muestre un diagrama de flujo para observar el progreso del diseño y el desgaste de la muestra.

Estas características del ECA en la revisión de los 66 artículos se resumen en la siguiente tabla:

Tabla II. Características principales de los Ensayos Clínicos Aleatorios analizados. Puebla, México, 2014.

\begin{tabular}{lcccc}
\hline Características & \multicolumn{2}{c}{ Si cumple } & \multicolumn{2}{c}{ No cumple } \\
\cline { 2 - 5 } & $\%$ & $\mathbf{f x}$ & $\%$ & $\mathbf{f x}$ \\
Aleatorización & $89.3 \%$ & 59 & $10.6 \%$ & 7 \\
Grupos paralelos & $83.3 \%$ & 55 & $16.7 \%$ & 11 \\
$\begin{array}{l}\text { Descripción de las } \\
\text { intervenciones del grupo(s) }\end{array}$ & $57.6 \%$ & 38 & $42.4 \%$ & 28 \\
control & & & & \\
Enmascaramiento & $28.8 \%$ & 19 & $71.2 \%$ & 47 \\
Diagrama de Flujo & $54.5 \%$ & 36 & $45.5 \%$ & 30 \\
\hline
\end{tabular}

\section{DISCUSIÓN}

El presente estudio tuvo como finalidad evaluar si los ECAS realizados por enfermeras en los últimos siete años cumplen con la rigurosidad metodológica establecida por los criterios de la Declaración CONSORT.

\section{Características generales del estudio}

Autoría. La mitad de los ECAS analizados tienen como primer autor a un doctor en ciencias, lo que es congruente dada la formación en este grado académico cuyo principal objetivo es facilitar la transición del conocimiento a la práctica a través de la manipulación de variables ${ }^{(13)}$. 
Temática. La temática que prevaleció fue la clínica. Parece lógico pensar que este tipo de diseño tiene mayor factibilidad en los hospitales que en las intervenciones en la comunidad o en familias. Este hallazgo es similar a lo reportado por García Rodríguez ${ }^{(12)}$, donde las variables más utilizadas fueron de tipo clínico, lo que indica que las evaluaciones incluidas en los ECAS tienen claro interés y repercusión sobre los pacientes del ámbito hospitalario ${ }^{(14)}$. Sin embargo, se requiere de mayor presencia en la publicación en revisitas indizadas y con buen factor de impacto, dado que las publicaciones de ECAS por enfermeras son escasas, la figura de la enfermera de ensayos clínicos no está claramente descrita y permanece como un ente desconocido para la mayoría de los profesionales que trabajan en un hospital ${ }^{(11)}$.

Investigadores.La mayoría de los ECAS en este estudio no incluyen otras disciplinas diferentes a Enfermería, cifras que coinciden con los hallazgos de García Rodríguez ${ }^{(12)}$, donde la minoría de los artículos no muestran colaboración multidisciplinaria, pero la situación es similar en otras disciplinas ${ }^{(7,8)}$. En este sentido creemos que para afrontar los problemas de estudio en ECAS, necesariamente los investigadores deben pertenecer a varias disciplinas que puedan contribuir a clarificar el fenómeno de investigación.

Título y Resumen. La mayoría de los trabajos evaluados no facilitó la búsqueda, dado que en el título y resumen se debe identificar como un Ensayo Clínico Aleatorizado como lo marca la Declaración CONSORT, sin embargo, pudieron ser incluidos porque en la sección de metodología se mencionaron que se trataba de ECAS. Si se quisiera hacer una revisión sistemática o meta-análisis de una temática en particular que haya sido abordada como Ensayo Clínico Aleatorizado, la simple situación de no mencionarse en el título puede ser motivo de exclusión, aunque metodológicamente estén bien estructurados.

Antecedentes. Uno de los lineamientos que se evalúan en cualquier trabajo de investigación es referir los estudios previos, en nuestra evaluación, la mayor parte de los artículos lo mencionan, es claro que los artículos que no muestran antecedentes en sus investigaciones carecen de poder realizar conjeturas neutrales al comparar sus grupos paralelos, por lo tanto en estos artículos, los resultados no dan la seguridad de que las intervenciones o tratamientos sean confiables, situaciones que no solo se dan en la disciplina de enfermería y es bien conocida por los informes publicados en otro tipo de revistas ${ }^{(9)}$.

Prueba piloto.Una minoría menciona haber aplicado prueba piloto, esto es respaldado por el informe de García Rodríguez y Abeille Mora donde la mayoría de los estudios experimentales no expresan un entrenamiento previo o lo omiten ${ }^{(12,15)}$, sin embargo, este resultado debe considerarse con reserva, porque es posible que la prueba piloto no fue necesaria dado que los instrumentos ya han sido validados en estudios previos o los investigadores son expertos, por lo que simplemente no lo mencionaron.

Financiamiento. En el caso del financiamiento (de material o equipo), la mayoría de los ECAS no especificaron la fuente de financiamiento ${ }^{(7)}$. Situación que permite la subjetividad, posiblemente son investigaciones con financiamiento de los propios investigadores, de cualquier forma la fuente de la partida presupuestal no es clara. 


\section{Aspectos Metodológicos.}

Selección de participantes. La mayor parte de los artículos analizados presentan criterios de selección, disminuyendo variables confusoras. Sin embargo los criterios de eliminación en poco más de la mitad de la muestra no se refieren, coincidiendo con las puntuaciones realizadas por González Barahona ${ }^{(16)}$ quien menciona no detallar los criterios de exclusión, representa un sesgo que influye en la validez externa de los resultados.

Equivalencia y aleatorización. Más de la mitad de los ECAS analizados muestran tablas de características basales- demográficas, los resultados concuerdan con el reporte de Manríquez ${ }^{(8)}$, de 66 ECAS, el $80.3 \%$ de los artículos informó claramente sobre las características demográficas, lo que facilita observar si los grupos son equivalentes, mostrando aparentemente una correcta aleatorización. Sin embargo, en nuestra evaluación, la mayor parte de los ECAS no mencionan el método para generar la secuencia de asignación aleatoria, no garantizan conocer que los grupos son equivalentes, resultados similares con el reporte de García Alamino ${ }^{(7)}$, donde menciona que la exposición al tratamiento o intervenciones tal vez no fueron equiparables.

Intervenciones. La mayoría de los ECAS analizados detallan las intervenciones y especifican que tuvieron una variable respuesta después de la intervención; esto permite conocer cuáles fueron los cambios desde el punto de vista estadístico que se obtuvieron, sin embargo otros estudios similares, hallaron que en los estudios analizados no se detallan los procesos y análisis ${ }^{(7,8)}$.

Tamaño de la muestra. Ya ha sido evidenciado que la mayoría de los trabajos no clarifican el método para calcular la muestra ${ }^{(8)}$, tal es el caso de nuestro estudio, situación que disminuye el poder estadístico que puede llevar a una conclusión errónea de que no hay diferencias entre los grupos paralelos, destacando el error tipo I o alfa; dado que un tamaño de muestra pequeño da respuestas fiables a las preguntas formuladas o hipótesis de estudio que necesitan ser probadas. Por el contrario un tamaño de muestra grande puede hacer que el estudio sea difícil de manejar, gastar tanto tiempo y esfuerzo y es esencial para la producción de resultados de investigación útiles ${ }^{(17)}$.

Número de mediciones de la variable principal. La mayor parte de los ECAS no realizó medición intermedia de las variables, dado que la mayoría fueron diseñadas bajo un modelo test, re- test. Sería importante tener una comparación entre cada una de estas para observar la evolución del fenómeno, tener más control sobre la variable que se está manipulando y observar los cambios que se van obteniendo, pero esto depende de varios factores, tanto del diseño del estudio como del financiamiento para realizar mediciones repetidas.

Ciego. En experimentos, las intervenciones ciegas o enmascaradas son importantes para evitar sesgos y realizar comparaciones más certeras; en nuestra revisión, gran parte de los ECAS no mencionan el enmascaramiento, por consecuencia es posible que las comparaciones entre los grupos paralelos sean imparciales, ya sea deliberadamente o inconscientemente, ocasionando una contaminación de la muestra que conlleva a un sesgo en el análisis final, esto concuerda con Manríquez ${ }^{(8)}$ y Flores Pineda $^{(14)}$, quienes encontraron que no se especifica claramente cómo se enmascararon a los participantes de los estudios. 
Diagrama de flujo. La progresión del estudio no fue mostrada esquemáticamente en más de la mitad de las investigaciones, como lo menciona la Declaración CONSORT, publicarlo permite observar la manera en que se va desgastando la muestra a través de la pérdida de sujetos para conservar la potencia estadística, si hubo intención de tratamiento o comprender el análisis de los resultados. La literatura revisada, no hace evidente la evaluación de este parámetro del CONSORT en el análisis de los ECAS en otros estudios.

Consideraciones éticas. Más de la mitad de los ECAS evaluados no mencionan haber utilizado un consentimiento informado por escrito. Sin embargo la obtención de un papel firmado no es garantía de que el participante haya comprendido los objetivos de la investigación ${ }^{(19)}$, esta situación podría dar mayor credibilidad si el protocolo hubiese sido registrado y evaluado ante un comité de ética, dado que la responsabilidad del mismo es asegurar la protección de los derechos, la seguridad y el bienestar de los posibles participantes del ensayo ${ }^{(18)}$, sin embargo gran parte de los trabajos evaluados no mencionan el registro ante un comité de ética, situación que debería ser exigida por las revistas especialmente en este tipo de diseños.

Limitaciones. La mayoría de los ensayos evaluados no mencionan las limitaciones que ocurrieron en el desarrollo de su estudio, sin estos datos no se permite realizar replicas mejoradas de los protocolos, dado que se permitiría establecer algunos de los factores que actúan como impedimento y reducen la aplicabilidad clínica del estudio ${ }^{(19)}$.

\section{CONCLUSIÓN}

De forma general, los ECAS publicados por enfermería en los últimos siete años no cumplen con los criterios de la Declaración CONSORT. Los resultados pueden considerarse como limitaciones metodológicas del diseño y estadística que no contribuyen a presentar una evidencia confiable.

Esto debe considerarse justo ahora que es reciente el trabajo con diseños experimentales en enfermería. Si los ECAS se realizan con la confiabilidad estadística pertinente y con calidad en los procedimientos, podrían representar una clara evidencia científica en el futuro de las intervenciones de enfermería.

Estos hallazgos representan un área de oportunidad para que editores de revistas de enfermería soliciten a los autores mayor apego a la rigurosidad metodológica en sus artículos de acuerdo a los criterios de la Declaración CONSORT.

\section{REFERENCIAS}

1. Manterola C, Otzen T. Estudios Experimentales $1^{\text {a }}$ Parte. El Ensayo Clínico. Int. J. Morphol. 2015:33(1):342-349.

2. Cobos Carbò A, Augustovski F. Declaración CONSORT 2010: actualización de la comprobación para informar ensayos clínicos aleatorizados de grupos paralelo. Med Clin.2011: 137(5): 213-214.

3. Pérez Fuillerat N. Enfermería Basada en Evidencia y Transferencia de Conocimiento. Index de Enfermería.2015: 24(1-2): 5-6.

4. Cascaes da Silva, Valdivia BA, da Rosa R, Barbosa Gutierres PJ, da Silva R. Escalas y listas de evaluación de la calidad de estudios científicos. Revista Cubana de 
Información en Ciencias de la Salud [revista en internet]. 2013[citado 2016 agosto 31];24(3):295-312.

5. CONSORT.[Internet]: Ottawa: The Ottawa Hospital Research Institute; c2014 [consultado 05 mayo de 2014]. Disponible en http://www.consort-statement.org/.

6. González de Dios J, González Muñoz M, Alonso Arroyo A, Aleixandie Benavent R. Comunicación Científica (XIX) conocimientos básicos para leer (y escribir) un artículo científico (6): Listas de comprobación de estudios experimentales. Acta Pediatr Esp.2015:73(1):19-24.

7.García Alamino JM, Parera A, Olle G, Bonfil X. Características y calidad metodológica de los ensayos clínicos publicados en la Revista Española de Anestesiología y Reanimación. Rev. Esp. Anestisiol. Reanim. 2007: 54:333-339.

8. Manríquez J, Valdivia G, Rada G, Letelier LM. Análisis crítico de ensayos clínicos randomizados publicados revistas biomédicas chilenas. Rev. Méd. Chile. 2005: 133:439-446.

9. Alfonso F, Segovia J, Heras M, Bermejo J. Publicación en ensayos clínicos en revistas científicas: consideraciones editoriales. Rev. Esp. Cardiol. 2006:59(11):12061214.

10. Cárdenas Becerril L. Desarrollo del Pensamiento Reflexivo y Crítico. Ciudad de México: Cigome, 2014.

11. Guerrero Molina A, Millán Vázquez G, Cruzado Álvarez C, Medina Fernández

M. La enfermera de ensayos clínicos en el ámbito hospitalario: una figura desconocida. Rev. Cardiocore. 2013:48(2):75-78.

12. Garcia Rodríguez MG, Gómez Ávila MG, Aguilar Pérez I, Pérez Martínez GP, Velázquez Díaz L, Soriano Sotomayor $M$, Landeros Olvera E. Tendencias y características de la investigación en enfermería publicada en México. Rev. Enf Univ ENEO-UNAM. 2011: 8(1): 7-16.

13. Scaular Judith M. LA IMPORTANCIA DEL DOCTORADO EN CIENCIAS DE LA ENFERMERIA. Rev haban cienc méd [Internet]. 2008 Sep [citado 2016 Sep 05] ; 7( 3 ):. Disponible en: http://scielo.sld.cu/scielo.php?script=sci_arttext\&pid=S1729519X2008000300001\&lng=es.

14.Flores M, Márquez V, Candido A, Rodríguez J, Soriano M, Landeros E. Diseños Experimentales: ¿La Ciencia del Cuidado está Preparada? Rev. Horiz Enferm. 2015: 26(1):21-28.

15. Abeille Mora E, Soto Carrasco AA, Carrera Huerta S, Muñoz Muñoz VP, Sánchez Salinas R, Pérez Noriega E, Landeros Olvera E. Características de la Prueba Piloto: Revisión de Artículos Publicados en Enfermería. Rev. Enf Neurol. 2015:14(3).

16“González J, Fernández A B, Lucio F J (2013) Análisis crítico de ensayos clínicos de preparaciones de medicina herbal china. Dianas 2(1): e20130304. ISSN 1886-8746 dianas.20130304 URI

17. Shapiro H. Aspectos éticos de los ensayos clínicos en los países en desarrollo. Rev. Hosp. Materno Infantil. 2002: 21(1): 43-44.

18 Karlberg J, Speers M. Revisión de ensayos clínicos: una guía para el Comité de ética. Hong Kong: board, 2010 marzo. ISBN 978-988-19041-1-9.

19. Donado Gómez JH, Higuita Duque LN, Castro Palacio JJ. Limitaciones más frecuentes de los ensayos clínicos con asignación aleatoria (ECA) en el área de medicina interna. Rev. Med. 2015:23(2):35-40.

ISSN 1695-6141

(C) COPYRIGHT Servicio de Publicaciones - Universidad de Murcia 